Abstract 93 Table 1 Baseline lupus factors influencing damage accrual in 99 lupus patients. Regression analysis

\begin{tabular}{|c|c|c|c|}
\hline & OR & $\begin{array}{l}95 \% \text { confidence } \\
\text { limits }\end{array}$ & $\mathrm{p}$ value \\
\hline \multicolumn{4}{|l|}{ New damage accrual } \\
\hline Intercept & 0.085 & - & 0.024 \\
\hline Cytotoxics use (yes) & 12.64 & $0.88-182.47$ & 0.062 \\
\hline SLEDAI-2K & 1.130 & $0.996-1.28$ & 0.057 \\
\hline Anti-NSPA positivity & 17.604 & $1.13-273.52$ & 0.040 \\
\hline \multicolumn{4}{|c|}{ New damage accrual excluding anti-NSPA } \\
\hline Intercept & 0.20 & - & $<0.001$ \\
\hline Cytotoxics use (yes) & 11.866 & $1.39-101.53$ & 0.024 \\
\hline SLEDAI-2K & 1.149 & $1.03-1.29$ & 0.016 \\
\hline
\end{tabular}

with final damage whereas SLEDAI-2K and cytotoxics associated with accrual damage. Models including anti-NSPA showed its impact on final and accrual damage. Cognitive deficit, depression and other autoantibodies were not damage predictors.

Conclusions Our findings support that disease activity and cytotoxic use are relevant lupus damage predictors. Cognitive dysfunction and depression do not contribute to damage accrual as may fluctuate in lupus patients. A potential influence of anti-NSPA antibodies on damage accrual is proposed.

Funding Source(s): FONDECYT grant \# 1160513 to LM and CONICYT Basal grant \# AFB170005 to AG

\section{DEVELOPMENT OF SYSTEMIC LUPUS ERYTHEMATOSUS SYMPTOMS IS ASSOCIATED WITH CIGARETTE SMOKING}

${ }^{1}$ Karoline Lerang*, 'Torhild Garen, 'Inge-Margrethe Gilboe, ${ }^{2}$ Jan Tore Gran. 'Oslo University Hospital, Rikshospitalet; ${ }^{2}$ University of Oslo

\subsection{6/lupus-2019-Ism.94}

Background Smoking is associated with the development of aCCP positive rheumatoid arthritis. The association between smoking and SLE is more uncertain. 1) We wanted to compare SLE patients smoking habits before symptom development with population controls.

Methods 306 SLE patients fulfilling ACR classification criteria from Oslo area were sent a questionnaire in 2010 regarding smoking. Antibody profiles were collected from medical records.

Year of first symptom was defined by the patient, or if the doctors time of the first SLE symptom was earlier, this year was used. Juvenile SLE was defined by start of symptom before 16 years of age. One pack year was 20 cigarettes daily in one year.

The proportion smokers in the Norwegian population were found online 2) and every SLE patient was matched with population controls for the year of the first symptom, age (number not available for every years of age, but 10 year groups) and gender.

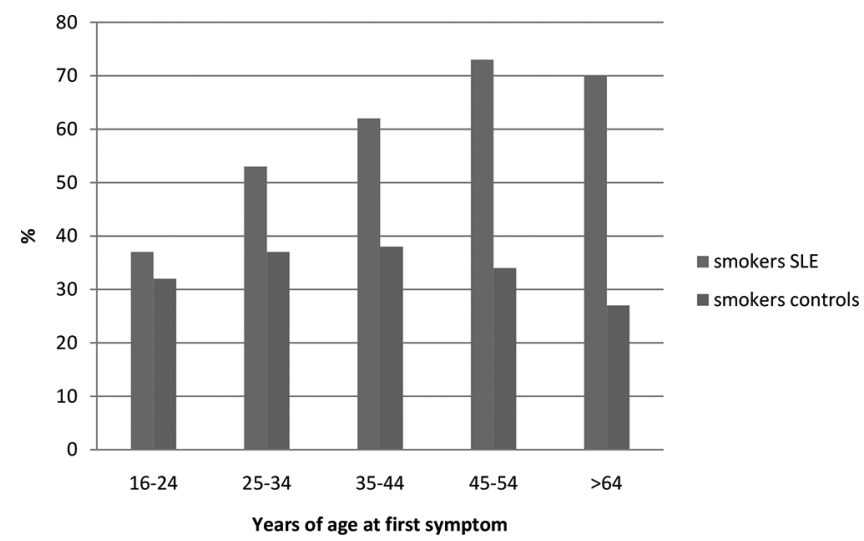

Abstract 94 Figure 1 The proportion of adult SLE patient smoking at the time of their first SLE symptom

Results 255/306 (80\%) SLE patients responded to the questionnaire. The mean age in 2010 was 48 years old (SD 15, min $18 \max 98)$ There were 39 patients with juvenile SLE whereof $64 \%$ were never smokers and $4 / 39$ (10\%) were smoking at the time of the 1 . symptom.

Among adult SLE, 116 (42\%) were never smokers and 83 (35\%) were previous smokers. In year 201053 (22\%) SLE patients were still smoking vs $20 \%$ in the control population (ns).

109/216 (50\%) adult SLE patients were smoking at the time of the first SLE symptom vs $35 \%$ in the matched control population $(\mathrm{p}<0,05)$, figure 1 . SLE patients had been smoking for a mean of 14 years when first symptom appeared and the mean pack year before first symptom were 10 (SD 11). The mean pack year after first symptom were 1 (SD 5).

Ever smokers did not have more dsDNA, aSm or aRNP antibodies, but they had a tendency to more discoid lupus (17\% vs $8 \%$ ).

Conclusions Even though prevalent SLE patients do not smoke more than their population controls, SLE patients are more often smokers at the time of the first symptom, especially in the higher age groups. This might indicate smoking as an environmental risk factor for developing SLE. However, smoking is tightly related to several other socioeconomic and cultural factors which must be explored in a prospective manner. Funding Source(s): None

\section{THE PERFORMANCE OF A RENAL ACTIVITY INDEX IN LUPUS NEPHRITIS IN INDUCTION THERAPY}

${ }^{1}$ Najla Aljaberi ${ }^{*},{ }^{2}$ Theresa Hennard, ${ }^{1}$ Arjun Mathur, ${ }^{2}$ Hermine I Brunner. ${ }^{1}$ Cincinnati Children's Hospital; ${ }^{2}$ Cincinnati Children's Hospital Medical Center

\subsection{6/lupus-2019-Ism.95}

Background Renal involvement in systemic lupus erythematosus (SLE) is associated with high morbidity and mortality. Current standard tools to monitor lupus nephritis (LN) are suboptimal compared to the invasive renal biopsy. The renal activity index in lupus (RAIL) was developed using 6 urinary biomarkers to reflect disease activity. In children this tool was $92 \%$ accurate in identifying active LN. We aim to study the changes in this score in relation to induction treatment in $\mathrm{LN}$. 